\title{
Development of a case study methodology in the information technology (IT) field in South Africa: a step-by-step approach
}

\author{
S. Lubbe \\ Department Information Systems and Technology \\ University of Durban-Westville, South Africa \\ slubbe@is.udw.ac.za
}

\section{Contents}

1. Overview of the study

2. Focus on key concepts

3. Aim of the research

4. Why research study is important

5. Nature of a methodology

6. Initial stages of research

7. Research options

8. Bias in research

9. Case studies as research strategy

10. Different types of evidence on which a case study may be based

11. Bias in the case study

12. Significance of uniformity when recording data

13. Formality of the case study research methodology

14. Case study protocol - research objectives and schedules

15. Summary and conclusions

16. References

\section{Overview of the study}

Case study methodology and the philosophy that underpin IT research are discussed in this article. It seems that a majority of IT research projects rely on a number of data collection methods. It should also be remembered that people do what they know, do what they have done, do what is easier and do what is rewarded. It is therefore important to explore new avenues in IT research by expanding the definition of data and thereby creating opportunities for discoveries. 
The focus of any IT research project should first be on the development of a research culture. Engagement in research is critical for developing excellence in teaching and expertise because through research it is possible to encounter uncertainty and through uncertainties the potential to develop. The importance of qualitative data and the construction of narratives as a key procedure in the development of theoretical conjectures and empirical generalisations or hypotheses are also emphasised. Remenyi and Williams (1995) therefore put the importance of paradigmatic thinking and research into perspective by contrasting the paradigmatic approach with narrative thinking and qualitative research. Although the authors advocate the importance of qualitative research in IT, they also recognise the distinguishing feature of modern science as the formulation of laws that capture the essential features of a problem and enable predictions into the future and about different situations.

\section{Aim of the research}

Any research should be aimed at realizing a goal and it should be covered in such a way that it must be clear to the readers of the research results that the designer of the research knew what he or she set out to achieve. The aim of research in IT could be:

1. to study and evaluate the approach by South African organizations to the problem in the field of IT that will be researched;

2. to develop a preliminary theory of good practice in the field to solve the problem;

3. to test this theory by reference to other enterprises and practitioners; and

4. thereby develop the theory into managerial guidelines.

\section{Why research study is important}

Although IT evaluation has only recently become an important issue, interest in the IT research area has grown over the last couple of years. Academics have been interested in this field since the 1970s. However, practitioners and consultants have taken an intense interest in this subject since the end of the 1980s. It is sometimes thought that this business interest was the result of general management being computer literate during the 1980s and as a result more inclined to demand explanations about the effectiveness of their organizations' information technology investments. It is clear from previous research (Butler-Cox Foundation 1990; Barua, Kriebel and Mukhopadhyay 1991) that many organizations are concerned with research issues in IT because they are requested by their chief information officers (CIOs) to use resources effectively. This is also in keeping with a general need to become more competitive (Daniels 1994; Daniels and Daniels 1993). Thus, more and more organizations will attempt to establish, formulate and implement the IT that will meet their organization's needs for efficiency and effectiveness (Earl 1992; Farbey, Land and Targett 1992, 1993).

Much of the available literature and the research that are reviewed focus on a calculation of a problem in IT without commenting or providing guidelines for IT managers who wish to address the problem they are experiencing in such a way that would best fit into their present IT system. It is, of course, not easy to identify factors in IT that will support organizational strategies. To establish a method on how organizations can do this will be of practical value to managers. Similarly, the selections of IT investment factors require detailed attention and advice on how managers could apply them. Conducting research into practical issues can be regarded as a matter that demands attention. 


\section{Nature of a methodology}

A scientific methodology should form the basis of any academic research project which claims to add something of value to the body of knowledge. Chadwick, Bahr and Albrecht. (1984) describe scientific methodology as a systematic observation of nature.

Einstein (1950) argues that:

'the object of all science, whether natural science or psychology, is to co-ordinate our experiences into a logical system.'

Einstein (1922) defined science as:

'...the attempt to make the chaotic diversity of our sense - experience corresponds to a logically uniform system of thought. In this system, single experiences must be correlated with the theoretical structure in such a way that the resulting co-ordination is unique and convincing.'

Chadwick et al. (1984) define method as a way of assessing the validity of ideas about reality and existence through systematic study and observation, together with the recording of observations and how they were obtained so that the resulting factors may be checked and modified by others. Smith (1990) notes that knowing how to use the tools and techniques of research does not in itself guarantee the effectiveness of any person in carrying out a scientific investigation. Remenyi (1996) state that science is just one human way of looking at the world of reality. This way of thinking suggests that science interprets and it is not neutral. However, scientific theories are open to endless revision and current scientific ideas are nothing more than human work in progress. Remenyi (1996) states that scientific methodology is a system of explicit rules and procedures upon which claims of knowledge are evaluated.

Methodologies are neither closed nor infallible and Lee (1989) points out that:

'...scientists have not yet settled among them on a single method of what science is, let alone how it should be researched.'

Scientific understanding proceeds by way of constructing and analysing models of the segments or aspects of reality under study. The purpose of these models is not to give a mirror image of reality, not to include all its elements in their exact sizes and proportions, but rather to single out and make available for intensive investigation those elements which are decisive (Baran and Sweezy 1970). Lee (1989) notes that it was argued that no field can potentially be a science unless its theories are cast according to a demarcation criterion.

A methodology may serve as a set of rules for reasoning whereby evaluation of facts can be used to draw inferences. Haug (1996) notes that the field of evaluation is not static. Remenyi (1990) notes that the use of a methodology infers some competence in logical reasoning. The researcher might be able to establish or verify some theories and these must be validated by some form of empirical evidence. The primary benefits of a scientific methodology are therefore that it:

- facilitates communication between scientists, enabling them to share experiences. It also makes replication of the research easier. Replication of research is always necessary to safeguard against unintentional errors as well as deception or fraud;

- ensures that an acceptable logical structure is being used. Scientific research requires both empirical observation and valid logical reasoning. The methodology is an articulation of valid logical reasoning. The rules of classification, definition, deduction and indirect sampling, if used, must be articulated in the methodology; and 
- institutionalizes conceptual frameworks for communication, rules of reasoning, procedures and methods for observance and verification. Methodology demands conformity. However, care must be taken that methodology does not hinder new discoveries and, by implication, scientific progress (Nachmias and Nachmias 1989).

Methodology is an issue facing the social scientist in conducting research in that it provides a basis on which the researcher may assert the validity of his/her findings. Scientific knowledge, according to Remenyi (1990), is knowledge provable by both reason and observance and methodology must address both issues in terms of logic validity and empirical verification. These two criteria are translated into a research process.

\section{Initial stages of research}

The theoretical and philosophical basis of any study is empirical research. The research strategy that could be employed is based on the approach described by Remenyi and Williams (1995) (Figure 1). A literature survey of IT issues to be researched should be completed in two stages. After the first phase the literature review should be recorded because this becomes the basis of the research proposal which should be validated by presentation to a research committee for acceptance. In the second phase, the literature review can be extended, a critique developed and research questions established.

If there is no appropriate grand theory from which to derive hypotheses it can be decided to use a grounded theory approach and to collect evidence through the use of case studies. Thus case studies could be used to develop a theory or theoretical conjecture concerning the formulation, adaptation and implementation of IT and its benefits. Case studies can be used if the focus of the study is to understand the how and why of a phenomena of IT within the organizational context. The evidence collected through the empirical case study normally results in a large transcript, which could be analysed using both content and correspondence analysis, and thus develops into a grounded theoretical conjecture. The grounded theory can be cross validated by discussion with a focus group consisting of IT experts in practice and academia.

A focus group can thus confirm that the theory in IT was relevant and practical. A second level of validation could be undertaken by presenting the theory to several senior IT executives from leading organizations. These executives should be asked to confirm whether they could make use of the theory (action research). The research can thus employ a combination of qualitative and quantitative methodologies in order to ensure that it complies with scientific principles.

In summary, the research project could consist of three phases: case studies, a focus group and presentation of the results to practitioners for cross-validation purposes. It is believed that several case studies are needed to establish a useful theory. The second phase of the study, using the focus group, should be done to comply with the principle of falsifying, where an attempt is made to disprove the theory deduced from the case study work.

Figure 1 Research process derived from Remenyi and Williams 1995 


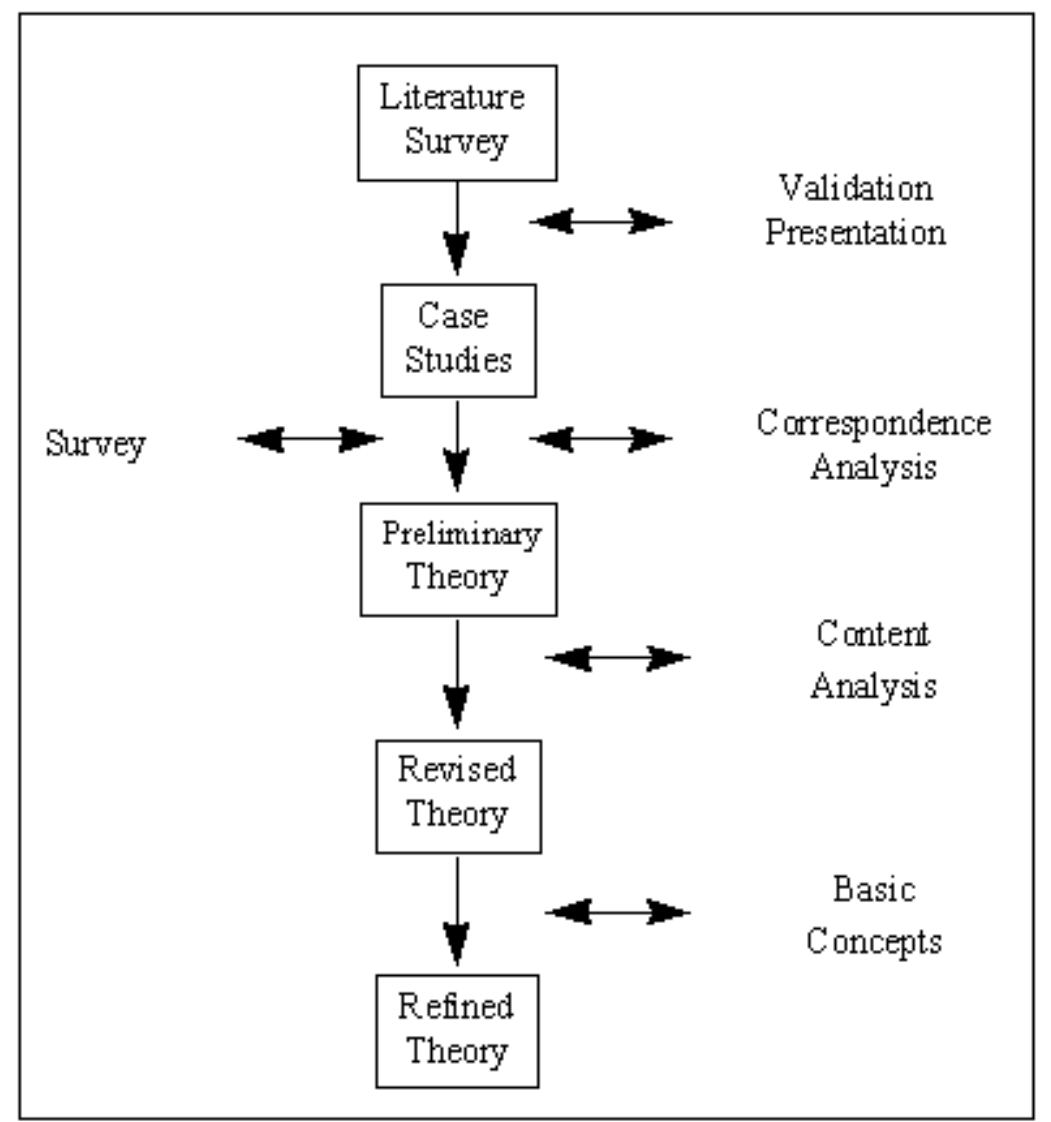

\section{Research options}

The researcher could decide to employ empirical research in the study because it would enable him or her to gain insight into the practical situations that exist around IT. Empirical research also helps the researcher to consider the meaning of certain naturally occurring phenomena in the world of IT (Easterby-Smith, Thorpe and Lowe 1993). On the other hand, research also has a theoretical underpinning as reflected in the literature. The theory produced could also be the result of reflections on ideas supplied by individuals consulted during the research.

\section{Bias in research}

On the issue of bias, it is naive to assert that any form of research, or perhaps human activity generally, is without bias. Even in the physical and life sciences the researchers' bias is reflected in the subject researched, the experiments chosen, as well as the way the experiment is conducted. Thus bias cannot be totally eliminated but should be recognized and its implications acknowledged and accepted. Sub-conscience bias on the part of the researcher is a problem. Of course triangulation may be used to help in this respect, but in the final analysis an argument based on a judgement is always required in research (Collins 1994). This may not be easy to make when personal prejudices play an overtly influential and important role. The bias problem has been described by Hubbard (1979):

'The mythology of science asserts that with many different scientists all asking their own questions and evaluating the answers independently, whatever personal bias creeps into their individual answers is cancelled out when the large picture is put together. This might conceivably be so if scientists were women and men from all sorts of different cultural and 
social backgrounds who came to science with very different ideologies and interests. But since, in fact, they have been predominantly university-trained white males from privileged social backgrounds, the bias has been narrow and the product often reveals more about the investigator than about the subject being researched.'

With regard to research findings, it is important that these are honestly presented and not produced in such a way as to simply support the opinions or prejudices of the researcher. Ideally the researcher is trying to apply 'disinterested intellectual curiosity' (Trevelyan 1942), but this is almost always impossible, as pointed out by Gould (1980):

'Science is not an objective, truth-directed machine, but a quintessentially human activity, affected by passion, hopes, and cultural biases. Cultural traditions of thought strongly influence scientific theories.'

Sometimes, if not frequently, personal bias is so subtle that the researcher is not even aware of it. In fact, many would argue that a researcher should not attempt to compensate for this bias, but should simply state clearly the possible biases involved and allow the readers to compensate for them.

\section{Case studies as research strategy}

The case study methodology's approach is about gathering data with which to develop grounded theory. It can be selected for several reasons. These reasons will become clear from the description below of the case study's methodology. The term 'case study' refers to two entirely different issues. As a teaching-learning device, it is an effective and well-established technique for use in the classroom to simulate real-life situations. The way to handle case studies is similar to the way business issues are mostly handled. Classroom and syndicate groups provide the simulated business meetings in which the participant can learn both the skills of listening and presenting a point of view. The environment in which the case study is used helps participants to develop a degree of confidence in their judgement, as well as a degree of humility (Edge and Coleman 1986).

\subsection{Definition of a case study as a teaching-learning device}

From a teaching-learning point of view, a case study may be defined as a technique for presenting ideas or data primarily for the purposes of simulating discussion and debate. Case studies may address any subject area and may range from single page documents to lengthy tomes. It is one of the oldest ways of presenting information with - it' roots reach back to times when myths and fables were used as teaching devices (Remenyi 1990). The fact that the circumstances portrayed in these situations were fictitious did not in any way detract from their use as learning material. Therefore, in general, a teaching-learning case study needs not reflect a complete or accurate rendition of a real-life situation as its purpose is simply to present a framework for discussion and debate. From the case writer's point of view, the main attribute of teaching-learning case studies is that they are written in such a way that they will stimulate readers to identify problems and seek solutions.

\subsection{Definition of a case study used as a research methodology}

As a research strategy a case study is a technique for answering who, why and how questions. The use of multiple evidence enables the researcher to provide a convincing argument as an answer to the questions. It is not essential to the validity of the case study research method that a case study should be able to be generalized. In this type of research, generalization is not a central issue. The relevance of a case study is more important than its 
generality. When a case study is carried out both systematically and critically and aimed at the improvement of understanding, then it is relevant, and if publication of its findings extends or expands the boundaries of existing knowledge of the subject area, then it is a valid form of research. As the case study methodology can produce excellent results in the hands of a skilled investigator, its use is on the increase in most areas of the social sciences as well as in information research.

Stake (1994) identifies three types of studies using the case study method. In the first type, he identifies intrinsic case studies in which the study is undertaken because the researcher wants a better understanding of the particular case. Using the instrumental case study as the second example, a particular instance is examined to provide insight into an issue or refinement of theory. In the third example, he uses the term collective case study. In this type of study researchers may jointly study a number of case studies to enquire into the phenomenon, population, or general condition. The advantage is that case studies are frequently discussed and analysed and, subsequently, courses of action are agreed upon in groups.

From a research strategy point of view, the case study methodology is a way of establishing valid and reliable information or findings that add to the accumulated knowledge of the processes by which business and many other organizations functions. It is a research strategy for the social scientist in the same sense as experiments are a research strategy for the natural scientist. Yin (1994) defines a case study as:

'...an empirical inquiry that investigates a contemporary phenomenon within its real life context, when the boundaries between phenomenon and the context are not clearly evident, and in which the multiple source of evidence are used. It is particularly valuable in answering who, why and how questions in management research'.

According to Bell (1987), the case study methodology has also been described as an umbrella term for a group of research methods that have in common the decision to focus an enquiry around a specific instance or event. The philosophy behind the case study is that just by looking carefully at a practical, real life instance a full picture can sometimes be obtained of the actual interaction of variables or events. The case study allows the investigator to concentrate on specific instances in an attempt to identify interactive processes that may be crucial but which are transparent in a large-scale survey. Therefore, the aim of the case study is to provide a three-dimensional picture of the situation. It should illustrate relationships, corporate-political issues and patterns of influence within a particular context.

Case studies are being used to a larger extent by social science as both a research and a teaching vehicle, especially in IS research (Lee 1989). The scope of the case study is extensive, ranging from individuals and organizational groups to national policies or events. Cases are either persistent original (Stake 1994), or one-of-a-kind. Cases are compared and their characteristics are studied and behaviour patterns noted. It is important to bear in mind that most cases are aggregates of complex behaviour (Stake 1994). From a research point of view, the case study methodology describes the total situation as a combination of different factors. The case study may focus on the description of the process or sequence of events in which the behaviour occurs, the study of individual or group behaviour in its total social setting, or the comparison of cases leading to the formulation or confirmation of hypotheses, as claimed by Stake (1994).

By means of the case study method, it is possible to establish the number and variety of properties, qualities and habits combined in a particular instance. The depth of the inquiry possible through the case study method is greater than any other research method, such as a survey (Galliers 1991). For example, although attempts are made to ascertain attitudes by means of questionnaires, the results are sometimes unsatisfactory as one cannot do justice to 
an attitude by ticking yes or no, or rating something between 1 and 5 . The case study can go much further than this superficial analysis and can discuss greater variations and reasons for attitudes (e.g. if a respondent is asked to rate a service rendered in a case study context, he or she may reply with both a score on a preset scale and a list of explanations and/or caveats qualifying the score). Additional information is beneficial to the researcher who may use it either as explanatory notes to the findings produced from the questionnaire or as raw material for content analysis.

Because the case study follows the logic of the experiment rather than logic of the survey, it is not necessary to repeat a case study (Yin 1994). This is because the experiment starts with the formulation of a theory and then attempts to find evidence that will either support or disprove the theory, while the survey attempts to get a general view of something. The experimental approach is used in the case study method of research. The reason why it is not necessary to repeat the experiment or case study many times, as with a survey, is the same as why it is not necessary to experiment with the boiling point of water many times to support or contradict the theory of transformation of liquids into gasses at a certain temperature. However, it is important to bear in mind that the case study methodology is not designed to measure the frequency of occurrence of events but rather to support or reject theoretical propositions.

\section{Different types of evidence on which a case study may be based}

From a case study research strategy point of view, the case study methodology implies comprehensive and intensive study of the subject. Thoroughness is therefore one of the first pre-requisites. Facts must be ascertained from the enterprises or enterprises under study and then carefully interpreted. These may be obtained from documents, archives, interviews with any person who has knowledge of the subject, observations of the investigator, participantobserver interaction, as well as physical artefacts. This information must be weighed, tested and sifted to eliminate fictitious and false statements as well as, where possible, personal opinions.

\section{Bias in the case study}

Case studies can rarely be completely objective due to the bias of both the supplier and the recipient of information. Stake (1994) regards this area of research as fraught with danger, primarily due to the problem of subjectivity when interpreting data after they have been written down. Bias is everywhere, but can be minimized. It is the primary function of the researcher to minimize the bias level in which he or she is working. There are at least three obstacles in obtaining unbiased testimonials from observers:

1. Difficulties encountered by individuals to remember accurately

2. Inhibitions of individuals to disclose important feelings

3. Suspicion of individuals about revealing information that might reflect poorly on them or their superiors.

The use of multiple sources of evidence, however, can help substantially in improving the validity and reliability of the research. By studying every aspect of the problem from as many angles as possible, and by using various sources of evidence, the case study research strategy is a powerful research tool in the hands of a skilled investigator (Stake 1994). 


\section{Significance of uniformity when recording data}

In multiple case study research, where a series of IT cases are involved, uniformity of recording should be sought as it facilitates comparison between enterprises and situations that allows similarities and differences to be highlighted. Unless there is some uniformity, it can be extremely difficult to recognize similarities, and much of the usefulness of the case study method, as well as its scientific value, may be eliminated. The case study methodology presumes a well-defined problem with a clearly articulated hypothesis. This is part of case study design and it is necessary to have this aspect of the research in place before an attempt is made to collect evidence or perform analysis.

\section{Formality of the case study research methodology}

The case study research methodology is often mistakenly thought to be informal (Stake 1994). This is because it is confused with case writing from a teaching-learning point of view. In fact, the case study research strategy methodology requires a distinctly formal, structured approach. Before the interviewees can be approached, protocols must be drawn up. The protocol is a formal and detailed master plan for IT research. It is a document in which full details of the case study research design - including details of the questions to be asked, field procedures for the researcher and details of all types of evidence required as well as the structure of the final research must be specified.

\section{Case study protocol - research objectives and schedules}

The case study protocol sets out both the instrument with which the case study is conducted and the general rules and procedures with which the work is carried out. The overview includes the objectives or issues on which the case study investigator should focus. Figure 2 displays the overview, as well as an interview schedule that can be used to collect data during unstructured IT case study interviews. The investigator has to work in the real world and thus cope with real world situations during the data collection plan, including the possibility of the respondent dropping out of the experiment or case study. Similarly, corporate documents may not always be available. The protocol for IT research is primarily a tactic for increasing the reliability of the case study procedure and needs to emphasize issues such as:

- Defining who should be interviewed

- Gaining access to the right people

- Having adequate resources available such as time, paper, tape records, etc.

- Developing a procedure for discussing the research with other researchers

- Making a schedule of the required data collection activities, providing contingencies.

A protocol could have the following sections:

\section{Objectives of the research}

The main objective of the case study research is to obtain evidence on how firms formulate and implement IT and how to link IT to organizational performance. 
This will be achieved by using a series of unstructured interviews which will allow
informants the opportunity of supplying information on a wide range of issues
related to information, decision making and implementation activities, and
organizational performance. It is intended to allow the informants as much
freedom in the interviews as possible as it is crucial to ensure that the interviewer
does not in any way prejudge the evidence offered by them. Nonetheless, a list of
discussion topics that the interviewer may use as an interview schedule has been
developed. The topics are available to assist the interviewer if the discussion
requires some prompting or guidance.
Key issues of the research
There are four key issues of this research:
1. To establish how managers formulate IT, that is, are highly systematized
methodologies used or do the organizations respond to other stimuli?
2. To discover what is involved in implementing sound IT, for example are
they implemented in much the same way as other investment decisions or
are there any significant differences?
3. How does an organization handle threshold decisions on IT?
4. How do they link IT to organizational performance?
11. How do they determine whether IT has an effect on organizational
performance?
Interview schedule
10. Have operators and managers received appropriated training?
they the IT department have greater freedom to respond to exceptions when
1. How does the organization categorize IT?
2. What architecture does the hardware consist of?
3. Does top management agree with the definition and compilation of IT in the
organization?
4. Has the IT been tied to any other investment decisions?
been documented in specific detail including costbenefit or other types of
financial analysis?

Figure 2 Example of research objectives and interview schedules

\subsection{Case study protocols}

At the centre of the protocol is a set of questions reflecting the IT enquiry. There are some characteristics that distinguish such a set of questions from those used in a survey. First, the protocol questions are set for the investigator and not the respondent. The questions are reminders or prompts to the investigator concerning the information that has to be collected. Second, each question should be accompanied by a list of sources of evidence that cover observations and interviewees' comments. It is important to remember that a particular protocol is designed for data collection from a single case and is not intended to serve the 
entire project. Therefore, in multi-case situations in IT, a number of protocols will be required.

\subsection{Guide to what is required for the case study report}

The guide to compiling the case study report forces the investigator to think about the audience for which the case study is intended early in the process. The investigator should be concerned throughout the study with the design of the final report. An outline of the case study report should be included in the case study protocol. The protocol should indicate to what extent documentary evidence will be used in the final report. Case studies often produce large amounts of documentation and this may be used to produce an annotated bibliography. The bibliography may be helpful to readers in suggesting what is available for further research. Figure 3 shows the case study report guidelines.

\section{Case study report guidelines}

The following are the primary headings that were established as the key focal points of the case study reports. These were established earlier in the research process so that they could be used by the author as a supplementary aide memoir in conducting unstructured interviews with informants.

1. Introduction and general background of the organization.

2. The state of IT within the organization.

3. The reasons for the current IT decisions.

4. The implementation of the IT decision.

\section{Figure 3 Case study report guidelines}

Using a well-defined protocol, the case study method complies with the basic tenets of scientific method as it involves classification, observation and descriptions of sequences and consequences.

It is a misunderstanding to claim that the case study method is by nature qualitative. In fact, case studies are highly quantitative. Wherever possible, purely descriptive data should be converted into quantitative data and statistical techniques applied. In addition, it is possible to conduct a survey within a case study situation and to use quantitative techniques in the analysis of data so collected. It is worth noting that qualitative data today is quantitative tomorrow. Quantitative and qualitative techniques are not conflicting but rather are complementary. There will, however, always be qualitative data that cannot be quantified. Although some situations may appear to be similar, they are in fact unique and statistical comparisons and correlations may actually lead to false assumptions and possibly to wrong conclusions. With regard to quantitative and qualitative research, it is important to remember that even in the physical sciences there are several disciplines (such as botany and zoology) that are primarily non-quantitative.

\section{Summary and conclusions}

In conducting this research study, a considerable amount of time and attention was devoted to the issue of methodology because the author sees this as the foundation on which the credibility of case study research stands. Further, as articles are primarily an apprenticeship in research, the author believes that as many different approaches to the research problem should be applied as possible. This belief is reinforced by the lack of universally accepted methodologies in research into information systems. 
Some of the methods that are used in this research rely on qualitative information, while others are based on highly quantitative approaches. The case study method was primarily used because it enabled IT to be examined in a holistic manner within a real life situation. Furthermore, the case study research strategy accommodates the belief in multiple methods of data gathering and data analysis. The focus group could be used to obtain access to a number of experts and thus to widen the range of views collected. It is generally believed that the more informants, the better. The focus group also provides an additional step towards triangulation. Finally, the presentation of the results to a number of leading consultants and practitioners could be performed to obtain further cross validation of the results.

\section{References}

Baran, P.A. and Sweezy, P.M. 1970. Monopoly capital: an essay on the American order. Harmondsworth, England: Penguin.

Barua, A., Kriebel, C.H. and Mukhopadhyay, T. 1991. An economic analysis of strategic information technology investments. MIS Quarterly 15(3):313-331.

Bell, J. 1987. Doing your research project: a guideline for first-time researchers in education and social science. Milton: Open University Press.

Butler-Cox Foundation. 1990. Getting value from information technology, Research Report 75. London: Butler-Cox Foundation.

Chadwick, B.A., Bahr, H.M. and Albrecht, S.L. 1984. Social science research methods. Englewood Cliffs: Prentice-Hall.

Cohen, M.R. and Nagel, E. 1984. An introduction to logic and scientific method. New York: Harcourt, Brace and World.

Collins, H. 1994. A broadcast video on science matters entitled 'Does Science matter'. London: Open University.

Daniels, N.C. 1994. Information technology: the management challenge. New York: Addison-Wesley.

Daniels, J.L. and Daniels, N.C. 1993. Global vision: building new models for the corporation of the future. New York: McGraw-Hill.

Earl, M.J. 1992. Putting IT in its place: a polemic for the nineties. Journal of Information Technology 7:100-108.

Easterby-Smith, M., Thorpe R. and Lowe A. 1993. Management research: an introduction. London: Sage.

Edge, A.G. and Coleman D.R. 1986. The guide to case analysis and reporting. London: System Logistics.

Einstein, A. 1922. The meaning of relativity. London: Chapman and Hall.

Einstein, A. 1950. The fundamentals of theoretical physics. In: Out of my later years. London: Philosophical Library. 
Farbey, B., Land, F. and Targett, D. 1992. Evaluating investments in IT. Journal of Information Technology 7:109-122.

Farbey, B., Land, F. and Targett, D. 1993. How to assess your IT investments: a study of methods and practice. London: Butterworth Heinemann.

Galliers, R.D. 1991. Choosing information systems research approaches in information systems research. New York: Alfred Waller.

Glaser, B.G. and Strauss, A.L. 1967. The discovery of grounded theory. Aldershot: Aldine Inc.

Gould, S.J. 1980. The Panda's thumb. London: WW Norton.

Haug, P. 1996. Evaluation of government reforms. Evaluation 2(4):417-430.

Hubbard, R. 1979. Have only men evolved? In: Hubbard, R., Henifin, M.S. and Fried, F. Women look at Biology looking at Women.

Lee, A.S. 1989. A scientific methodology for MIS case studies. MIS Quarterly 13:33-50.

Nachmias, C. and Nachmias, D. 1989. Research methods in the social sciences. London: Edward Arnold.

Remenyi, D. 1990. Strategic information systems, current practices and guidelines. Unpublished PhD dissertation. Henley-on-Thames: The Management College.

Remenyi, D. 1996. So you want to be an academic researcher in business and management studies! Where do you start and what are the key philosophical issues to think about? South African Journal of Business Management 27(1/2):22-33.

Remenyi, D. and Williams B. 1995. Some aspects of methodology for research in information systems. Journal of Information Systems 10:191-201.

Smith, N.C. 1990. The case study: a useful research method for information management. Journal of Information Management 5:123-133.

Stake, R.E. 1994. Handbook of qualitative research, edited by N.K. Denzin and Y.S. Lincoln London: Sage.

Trevelyan, G.M. 1942. British historian, English social history. New York: Columbia University Press.

Yin, R.K. 1994. Case study research: design and methods, 2nd ed. London: Sage.

\section{Disclaimer}

Articles published in SAJIM are the opinions of the authors and do not necessarily reflect the opinion of the Editor, Board, Publisher, Webmaster or the Rand Afrikaans University. The user hereby waives any claim he/she/they may have or acquire against the publisher, its suppliers, 
licensees and sub licensees and indemnifies all said persons from any claims, lawsuits, proceedings, costs, special, incidental, consequential or indirect damages, including damages for loss of profits, loss of business or downtime arising out of or relating to the user's use of the Website.

ISSN 1560-683X

Published by InterWord Communications for Department of Information Studies, Rand Afrikaans University 